\title{
Sechs Wege, die Kluft zwischen Lernen und Arbeiten zu überwinden
}

\author{
P. Robert-Jan Simons
}

\section{Einleitung}

„Transfer of learning occurs whenever prior-learned knowledge and skills affect the way in which new knowledge and skills are learned and performed“" (CORMIER \& HAGMAN, 1987, S. 1). In der Literatur geht es fast immer um jenen Transfer, mit dem die Lücke zwischen Training bzw. Unterricht und dem Arbeitsplatz (in seiner Einbettung in eine Organisation) geschlossen werden soll: Wie können wir Lehr-Lern-Umgebungen so gestalten, dass sie dazu beitragen, dass Transfer von einer (Lern-)Situation auf eine andere (Anwendungs-)Situation erfolgreich wird? Die Frage ist also, wie instruktionale Umgebungen zu gestalten sind, um eine bestmögliche Anwendung neuen Wissens und neuer Fertigkeiten im Arbeits- und Alltagsleben herbeizuführen. Die Transferdefinition von CORMIER und HAGMAN umfasst aber noch mehr; bei ihnen ist der Transfer von früherem Lernen sowohl auf neue Lernprozesse als auch auf Leistung und auf Wissensanwendung gemeint. Es gibt daher verschiedene Wege, wie Transfer zu Tage treten kann. Beispiele sind der Transfer von Vorwissen und Fertigkeiten auf neue Lernprozesse, oder der Transfer vom Erwerb neuen Wissens und neuer Fertigkeiten auf das Lernen am Arbeitsplatz (SIMONS, 1990).

Die anderen Transferfragen werden üblicherweise entweder ignoriert oder unter anderem Namen behandelt (etwa „Vorwissen“, „tacit knowledge“, „Vorbereitung auf lebenslanges Lernen"). Aus meiner Sicht sind die grundlegenden Transfermechanismen und -prinzipien für all diese Probleme jedoch ähnlich, wenn nicht sogar identisch. Mit anderen Worten: Transfer zielt auf verschiedene Lücken zwischen früherem Lernen und Vorwissen, neuen Lernprozessen und der Leistung am Arbeitsplatz ab. Wir können vier Situationen unterscheiden, nämlich (1) Lernen vor dem Training und der Arbeit, (2) Lernen während des Trainings, (3) Lernen am Arbeitsplatz und (4) Arbeiten. Es ergeben sich sechs Lücken, zu deren Überbrückung Transfer notwendig ist. In diesem Kapitel geht es darum, diese sechs Transferarten zu definieren und zu veranschaulichen. Abbildung 1 stellt sie im Überblick dar.

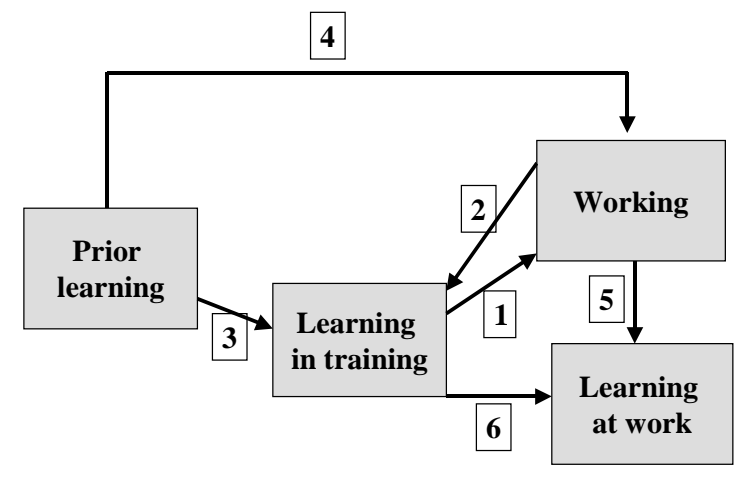

Abb. 1: Sechs Klüfte zwischen Lernen und Arbeiten. Kluft 1: Trainingstransfer. Kluft 2: Arbeit ins Training bringen. Kluft 3: Vom Vorwissen zum Lernen bzw. zum Training. Kluft 4: Anwenden von Kompetenzen. Kluft 5: Lernen am Arbeitsplatz. Kluft 6: Transfer vom Training zum weiteren Lernen am Arbeitsplatz. 


\section{Erste Kluft: Trainingstransfer}

In einer Reihe von Studien wurde bereits der Trainingstransfer behandelt (Abbildung 2; Kluft 1). Wie kann dieser Transfer unterstützt werden? Wie kann Trainingsteilnehmern beim Umgang mit Schwierigkeiten geholfen werden? Es gibt drei Maßnahmen: Maßnahmen zur Förderung weiten Transfers, Maßnahmen zur Förderung nahen Transfers, und organisationale Maßnahmen.

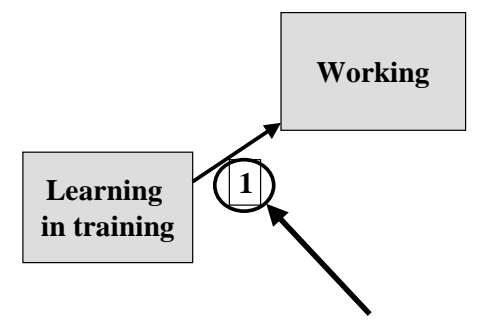

Abb. 2: Trainingstransfer (Kluft 1).

Die Literatur über Trainingstransfer konzentriert sich auf Maßnahmen zur Förderung des nahen Transfers. BRoAd und Newstrom (1992), ClaAs, POUwER und THIJSSEN (1986) sowie GainesROBINSON und ROBINSON (1989) kamen in Überblicksstudien zu dem Ergebnis, dass Prinzipien wie die in Tabelle 1 genannten der Trainingsplanung zu Grunde liegen sollten, wenn vor allem naher Transfer unterstützt werden soll.

Tab. 1: Prinzipien zur Trainingsplanung, die auf die Förderung naben Transfers abzielen.

\begin{tabular}{|c|c|}
\hline 1 & Explizite Lernziele sollten formuliert werden, die entweder auf nahen oder auf weiten Transfer abzielen. \\
\hline 2 & Diese Ziele sollten aus Bedarfsanalysen abgeleitet sein und mit der beruflichen Praxis zu tun haben. \\
\hline 3 & Die Ziele sollten so konkret wie möglich formuliert sein. \\
\hline 4 & $\begin{array}{l}\text { Die Ziele sollten auf die Eingangsvoraussetzungen der Lernenden abgestimmt sein bzw. an bestehende } \\
\text { Unterschiede adaptierbar sein. }\end{array}$ \\
\hline 5 & $\begin{array}{l}\text { Am Anfang eines Kurses sollte über die Ziele und Inhalte informiert werden, um falsche Erwartungen } \\
\text { korrigieren zu können. }\end{array}$ \\
\hline 6 & $\begin{array}{l}\text { Die Lerninhalte sollten so ausgewählt werden, dass sie sich auf konkrete (oder zumindest mögliche) } \\
\text { Arbeitssituationen beziehen. }\end{array}$ \\
\hline 7 & $\begin{array}{l}\text { Möglichkeiten, konkrete Inhalte zu verallgemeinern und Prinzipien aus ihnen abzuleiten, sollten gegeben } \\
\text { werden. }\end{array}$ \\
\hline 8 & Eine aktive Beteiligung der Lernenden wäre zu bevorzugen. \\
\hline 9 & Übungen zu allgemeinen Prinzipien sollten in unterschiedlichen Anwendungssituationen erfolgen. \\
\hline 10 & Lernende sollten angeregt werden, ihr Lernen selbst aktiv zu steuern. \\
\hline 11 & Lernende sollten anregt werden, ihr Lernen selbst zu überwachen. \\
\hline 12 & Die Bewertungskriterien für Lernleistung sollten klar sein. \\
\hline 13 & $\begin{array}{l}\text { Die Lernleistung sollte regelmäßig überprüft und bewertet werden, damit Feedback über den Fortschritt } \\
\text { gegeben werden kann und somit Wissenslücken identifiziert werden können. }\end{array}$ \\
\hline
\end{tabular}


Maßnahmen zur Unterstützung weiten Transfers bei der Gestaltung von Lernumgebungen zielen darauf ab, die Transferwahrscheinlichkeit von einer Lernsituation auf viele verschiedene Anwendungssituationen zu erhöhen. Hiervon ist also die Rede, wenn es nicht eine eindeutige Anwendungssituation gibt und wenn die Transferdistanz zwischen Lern- und Anwendungssituation relativ groß ist. Am Wichtigsten ist es dabei, die Verfügbarkeit von Gedächtnisrepräsentationen zu erhöhen. Tabelle 2 enthält die wichtigsten Möglichkeiten zur Unterstützung weiten Transfers (SIMONS, 1990).

Tab. 2: Prinzipien zur Trainingsplanung, die auf die Förderung weiten Transfers abzielen.

\begin{tabular}{||l|l||}
\hline \hline 1 & $\begin{array}{l}\text { Stärkere Vernetzung von Gedächtnisrepräsentationen, indem Beziehungen zwischen Konzepten expliziter } \\
\text { gemacht oder indem nur die Konzepte gelehrt werden, die besonders zentral sind. }\end{array}$ \\
\hline 2 & $\begin{array}{l}\text { Erhöhen der subjektiven Relevanz des zu lernenden Wissens und der zu erwerbenden Fertigkeiten, indem } \\
\text { Lernenden deren Nützlichkeit erklärt wird oder indem Lernenden die Aufgabe gestellt wird } \\
\text { herauszubekommen, worin ihre Relevanz liegt. }\end{array}$ \\
\hline 3 & $\begin{array}{l}\text { Bessere Vernetzung von Lern- und Arbeitskontexten, z.B. durch Lernen am Arbeitsplatz oder durch } \\
\text { Berücksichtigung praktischer Erfahrungen der Lernenden. }\end{array}$ \\
\hline 4 & $\begin{array}{l}\text { Verbesserung metakognitiver Fertigkeiten (direkt durch entsprechendes Trainingsmaßnahmen oder } \\
\text { indirekt durch die Gestaltung von Lernumgebungen). }\end{array}$ \\
\hline 5 & $\begin{array}{l}\text { Erhöhen der Verallgemeinerbarkeit von Wissen und Fertigkeiten durch Gelegenheit zur Reflexion oder } \\
\text { durch vielfältige Übungsangebote. }\end{array}$ \\
\hline \hline
\end{tabular}

Abgesehen von den beiden genannten Wegen, im Trainingsdesign selbst Transfer zu unterstützen, gibt es noch organisationale Maßnahmen (BALDWIN \& FORD, 1988; CLAAS et al., 1986). Diese sollen auf Organisationsebene zum Trainingstransfer beitragen. Einerseits geht es darum, das Handeln von Managern und Trainern $\mathrm{zu}$ beeinflussen. Andererseits gibt es Maßnahmen, die vor und nach dem Lernen zu treffen sind. Im Wesentlichen entsprechen solche Aktivitäten dem training for impact-Ansatz von GAINES-ROBINSON und ROBINSON (1989). Zunächst sind wichtige Entscheidungsträger ins Boot zu nehmen, die anerkennen, dass die Trainingsziele wichtig für die Organisation sind, und die wiederum andere relevante Personen davon überzeugen. (Natürlich muss gewährleistet sein, dass die Ziele tatsächlich wichtigen und aktuellen betrieblichen Bedürfnissen entsprechen.) Auf Organisationsebene muss zudem die richtige Auswahl von Trainingsteilnehmern getroffen werden, die das erforderliche Vorwissen besitzen und lernbereit sind. Ferner muss die Trainingsteilnahme durch ein entsprechendes Support-System am Arbeitsplatz gestützt werden, was etwa die Freistellung von der Arbeit für die Trainingsteilnahme beinhaltet. GAINES-ROBINSON und ROBINSON (1989) betonen zudem, wie wichtig es ist, dass nach dem Training innerhalb der Organisation gewürdigt wird, was gelernt wurde und inwiefern die Arbeitstätigkeit dadurch verbessert wird und was dies für das ganze Unternehmen bedeutet. Schließlich kann der Trainingstransfer durch Hilfe und Beratung bei der Integration des Gelernten im Betrieb unterstützt werden.

In einer Reihe von Studien in unserer Arbeitsgruppe wurde die Umsetzung solcher organisationalen Maßnahmen untersucht; die wichtigsten Ergebnisse sind folgende:

(1) Die Beteiligten unterscheiden sich erheblich in ihrer Einschätzung der Maßnahmen. KeRSTEN (1990) zeigte in einer Studie in psychiatrischen Kliniken, dass Studierende die Zahl transferunterstützender organisationaler Maßnahmen viel niedriger schätzten als ihre Ausbilder, diese wiederum niedriger als Manager und Berater. GEELEN und HENDRIKS (1998) kamen zu vergleichbaren Ergebnissen bei einer Versicherungsgesellschaft. Erfahrene Studierende beurteilten die Lage noch negativer als Anfänger. 
(2) Kersten (1990) sowie GEelen und HendriKs (1998) zeigten außerdem, dass die Beteiligten ihren eigenen Beitrag überschätzten: Sie waren mit ihrem eigenen Beitrag zur Unterstützung des Transfers zufriedener als mit dem anderer beteiligter Parteien.

(3) Im Durchschnitt werden viele der in den Tabellen 1 und 2 angeführten Prinzipien zur Trainingsplanung umgesetzt, aber nicht die genannten organisationalen Maßnahmen (GEELEN \& HENDRIKS, 1998; KERSTEN, 1990).

(4) Es wird mehr Gewicht auf Maßnahmen zur Unterstützung nahen Transfers gelegt als auf solche, die weiten Transfer fördern. TER HEIJNE und TERMOTE (1990) zeigten z.B. in einer Studie über ein Training für Kundendienstmitarbeiter in einem Diagnoselabor für Unterhaltungselektronik, dass zahlreiche Maßnahmen zur Unterstützung nahen Transfers vorgenommen wurden.

(5) Das Ausmaß, in dem Transfermaßnahmen ergriffen wurden, korrelierte signifikant positiv damit, wie adäquat theoretische Konzepte am Arbeitsplatz aufgegriffen wurden (TER HEIJNE \& TERMOTE, 1990).

(6) Das Ausmaß, in dem Trainingsergebnisse auf den Arbeitsplatz übertragen werden, ist oft erschreckend gering, wie z.B. LETHER (1997) zeigte. Sie analysierte einen Kurs für Versicherungsangestellte zur Erweiterung ihres Aufgabenfeldes; es ist wichtig zu wissen, dass diese ihr Geschäft üblicherweise telephonisch erledigen. Verkäufer für Haus- und Autoversicherungen mussten lernen, auch Reiseversicherungen verkaufen zu können. Das Training umfasste einerseits alles notwendige Wissen über solche Versicherungen, andererseits aber auch kundenzentrierte Umgangsweisen und Strategien zum cross selling (also, wie man einen Kunden anspricht, um ihm zusätzlich zur gewünschten eine andere Versicherung zu verkaufen, also z.B. eine Reiseversicherung, wenn er wegen einer Hausversicherung anruft). Es gab zwei Trainingsvarianten: Die eine Gruppe lernte wie üblich in einer Seminar- bzw. Klassensituation; in der anderen Gruppe gab es nur wenige Präsenzphasen, dafür arbeiteten die Teilnehmer viel für sich mit eigens entwickeltem Selbstlernmaterial. In einem Nachtest war die zweite Gruppe der ersten klar überlegen. Zusätzlich rief ein fingierter Kunde die Teilnehmer an und überprüfte, ob sie die cross sellingStrategie auch einsetzten. War dies nicht spontan der Fall, lieferte der Anrufer zusätzliche Hinweisreize (z.B. „Ich werde bald in Ferien fahren“). Sprach ihn der Verkäufer dann noch immer nicht auf eine Reiseversicherung an, fragte der Anrufer explizit danach. Die Ergebnisse des mysteriösen Anrufes sprechen eine klare Sprache: Fast keiner der 43 Verkäufer bot spontan eine Reiseversicherung an. Auch nach dem Hinweisreiz boten nur wenige eine solche Versicherung an; die meisten aber verbanden den fingierten Kunden mit der zuständigen Abteilung für Reiseversicherungen, anstatt zu versuchen, sie selbst zu verkaufen. (Die Selbstlerngruppe schnitt übrigens ein wenig besser ab.) Eine Analyse der Gründe für den geringen Transfer zeigte, dass die Verkäufer ihr neues Wissen und ihre Fertigkeiten nicht nutzten, weil sie sich unsicher fühlten, weil sie cross selling nicht wirklich mochten, weil keine entsprechenden organisationalen Maßnahmen vorgenommen worden waren, und weil sie unter großen Zeitdruck arbeiten mussten.

Organisationale Maßnahmen werden deswegen oft nicht ergriffen, weil die Trainer nicht genügend Einfluss besitzen. Ein alternativer Ansatz, der zur Zeit in unserer Gruppe untersucht wird, ist, ob Trainer ihre Trainees womöglich eher beeinflussen können als Manager in der Organisation (siehe Kluft 2). Dann könnten sie ihre Trainees mit Strategien wappnen, das Fehlen organisationaler Maßnahmen ausgleichen zu können, indem sie den Transfer vom Arbeitsplatz ins Training statt des Trainingstransfers leisten, also genau den umkehrten Weg einschlagen. 


\section{Zweite Kluft: Arbeit ins Training bringen}

Für den Transfer vom Arbeitsplatz ins Training (Abbildung 3; Kluft 2) gibt es verschiedene Möglichkeiten. Die bekanntesten Strategien sind problembasiertes Lernen, der Einsatz von arbeitsplatznaher Simulationen, Projektunterricht und Fokussierung auf authentische Probleme, die dem Arbeitsalltag der Trainingsteilnehmer entstammen.

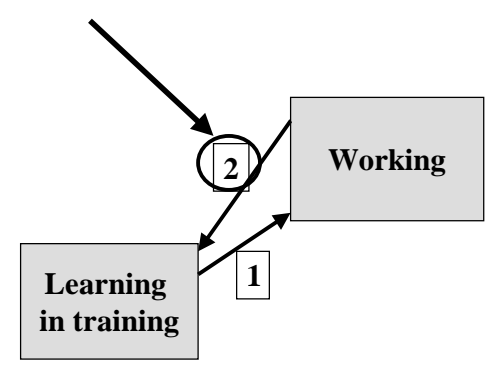

Abb. 3: Arbeit ins Training bringen (Kluft 2).

BRINKERHOFF und GILL (1992) sehen den zusätzlichen Wert eines Transfers vom Arbeitsplatz ins Training vor allem vor und nach einer Trainingsmaßnahme, weniger während des Trainings selbst. Die Grenzen zwischen Arbeitsplatz und Training verschwimmen, so dass sich die Teilnehmer während des Trainings auf ihre tatsächlichen Probleme am Arbeitsplatz konzentrieren können. Zwei in diesem Zusammenhang wichtige Strategien beim Design des Trainings sind nach BRINKERHOFF und GILL das Einnehmen einer Kunden- und einer Systemperspektive. Die Kundenperspektive einzunehmen bedeutet, dass die Trainingsteilnehmer und ihre Vorgesetzten als Kunden mit (geschäftlichen) Bedürfnissen, die erfüllt werden sollten, gesehen werden. Der Bildungsbedarf der Teilnehmer sollte gründlich erhoben, das Training ihren Wünschen gemäß gestaltet werden. Dies gilt nicht nur für die Teilnehmer selbst, sondern auch für all die Menschen, mit denen sie nach dem Training in ihrer Organisation wieder zusammenarbeiten müssen. Die Einnahme einer Systemperspektive bei der Gestaltung eines Trainings impliziert, dass Bezug auf die gesamte Organisation genommen wird, in der die Teilnehmer arbeiten, nicht nur auf Wissen und Fertigkeiten, die Gegenstand des Trainings im engeren Sinn sind. Vor welchem organisationalen Hintergrund, welcher Geschäftsstrategie und welchem Strukturwandel fiel die Entscheidung, dass die Teilnehmer zu der Trainingsmaßnahme entsandt wurden? Wie kann Lernen im Training zu diesen Veränderungen beitragen? Wer alles sollte einbezogen werden?

Ein alternativer Ansatz ist es, die Lernenden im Training zu wappnen, Lernen und Arbeiten besser als üblich zu verknüpfen. Ein Weg hierfür ist das Rückfall-Verhütungs-Training, bei dem die Teilnehmer verschiedene Strategien erlernen, um die Komponenten aus der Arbeit explizit in das Training einzubringen. Sie werden z.B. angeregt, die relevanteste der zu lernenden Fertigkeiten auszuwählen, sich nur auf ein spezifisches Element zu konzentrieren oder eine Strategie zu entwickeln, die ihnen dabei hilft, sich an das im Training Gelernte dann zu erinnern, wenn es am Arbeitsplatz gebraucht wird. Überdies werden sie angehalten, den praktischen Einsatz der gewählten Elemente am Arbeitsplatz im Auge zu behalten. In unserer Arbeitsgruppe wird derzeit ein anderer Zugang erprobt, bei dem Trainingsteilnehmer die Kluft zwischen Lernen und Arbeit dadurch überbrücken sollen, dass sie sich Arbeitssituationen bildlich vorstellen, in denen die gelernten Fertigkeiten angewendet werden können, aber auch Hindernisse, die einer Anwendung im Weg stehen. Dadurch wird den Teilnehmern bewusst, dass sie das im Training Gelernte oft nicht am Arbeitsplatz anwenden. 


\section{Dritte Kluft: Vom Vorwissen zum Lernen bzw. zum Training}

Die dritte Kluft thematisiert früher Gelerntes; es geht darum, wie Vorwissen beim Lernen und im Training genutzt bzw. nicht genutzt wird (Abbildung 4; Kluft 3).

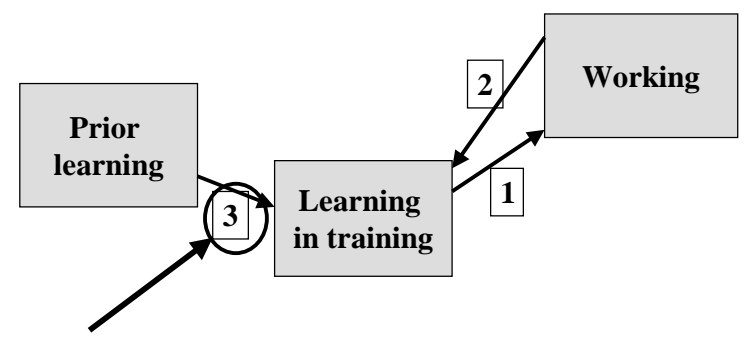

Abb. 4: Vom Vorwissen zum Lernen bzw. zum Training (Kluft 3).

Vorwissen kann in drei Zuständen auftreten: Es kann korrekt, inkorrekt oder teilweise korrekt sein. SIMONS (1999) postulierte, dass Lernende große Schwierigkeiten haben zu entscheiden, welches Vorwissen korrekt, inkorrekt oder nur teilweise korrekt ist. Überdies wissen sie aus Erfahrung, dass es manchmal unklug ist, Vorwissen bewusst ins Spiel zu bringen, weil dies zu Interferenz und Verwirrung führen kann. Einige Teile des Vorwissens werden aber automatisch aktiviert. Das größte Problem bei der Gestaltung von Lernumgebungen besteht in der Entscheidung, wie mit den Problemen Lernender umgegangen wird. Wie kann man ihnen dabei helfen, ihr korrektes oder teilweise korrektes Vorwissen zu nutzen, ohne dass es zu Interferenzen kommt, zugleich aber etwas gegen falsches (oder teilweise falsches) resistentes Vorwissen unternehmen?

Es gibt mittlerweile viele Studien, die auf vorwissensbezogene Probleme von Lernenden oder Lernumgebungen hinweisen, aber kaum Lösungsansätze. STRIKE und POSNER (1985) präsentierten ein Modell, das mit kognitiver Akkomodation arbeitet. Lernende sollten Unzufriedenheit mit ihrem Vorwissen, ihren Konzepten verspüren. Sie sollten lernen, neue wissenschaftliche Ansätze zu verstehen, die sich hinter neuen, zu erlernenden Konzepten verbergen. Die neuen Ansätze sollten ihnen natürlich dabei helfen, ihre Probleme zu lösen. Außerdem sollte ihnen Gelegenheit gegeben werden, ihre neuen Konzepte und ihr neues Wissen zu überprüfen und zu bewerten. PRAWAT (1989) betonte in diesem Zusammenhang die große Rolle metakognitiver Bewusstheit. „Students should be aware of their pre-conceptions before they will be able to restructure them. Students must first recognise that the new information is related to what they already know; they then have to link this information to two types of prior knowledge - that which is consistent with the scientific notions and that which is incompatible with those notions. It is the latter connection that leads to the realisation that their own ideas are not complete or satisfying explanations and that the scientific view is a more convincing and powerful alternative" (PRAWAT, 1989, S. 12 f.). Wie SIEGLER (1983) zeigte, reicht Bewusstheit allein jedoch nicht aus: Akkomodation impliziert immer auch Akzeptanz. Lernenden sollten die neuen Konzepte plausibel erscheinen. NussBaum und NOVICK (1982) schlugen die Verwendung von Schlüsselereignissen vor, die das Vorwissen und die alten bestehenden Konzepte der Lernenden auf den Prüfstand stellen. HEWSON und HEWSON (1984) empfahlen, die Lernenden dazu zu bewegen, altes und neues Wissen zu vergleichen und zu kontrastieren.

Ali (1990) und Biemans (1997; Biemans \& SimOns, 1995, 1996) entwarfen eine Unterrichtsstrategie (die CONTACT-Strategie), die Lernenden beim Umgang mit ihrem Vorwissen helfen soll. CONTACT besteht aus fünf Schritten. 
(1) Lernenden sollte dabei geholfen werden, sich ihr eigenes Vorwissen zu verdeutlichen (mit Hilfe von exposing events).

(2) Lernende sollten angeregt werden, ihr Vorwissen mit der neuen gelernten Information zu vergleichen.

(3) Lernende sollten aufgefordert werden, die „neue Idee“ in Worte zu fassen.

(4) Lernende sollten die neuen Konzepte auf ein konkretes Problem anwenden.

(5) Lernende sollte als Folge von (4) die Angemessenheit der neuen Konzepte bewerten.

Natürlich löst diese Strategie nicht alle oben erwähnten Probleme; sie ist bei Gegenstandsbereichen besonders geeignet, bei denen Lernende viele Fehlkonzepte haben, die durch Unterricht korrigiert werden müssen. Sie hilft nicht bei der Bewältigung metakognitiver Anforderungen (z.B. sich der Funktionen der verschiedenen Arten von Vorwissen bewusst zu werden oder die kritische Rolle von Fehlkonzepten zu erkennen). CONTACT kann nur zur Anwendung gelangen, wenn Wissen über häufig vorkommende Fehlkonzepte vorliegt.

In einer Reihe von Studien zeigten ALI (1990) und BIEMANS (1997), dass die CONTACT-Strategie die Lernergebnisse von 11-13jährigen Schülern signifikant verbessert. Die Studie von BIEMANS umfasste neun Unterrichtsstunden über physikalische Geographie, in denen Begriffe wie Äquator, Erdrotation, Kondensation, Regen, atmosphärischer Druck, Wind oder Wetter behandelt wurden. Die Lektionen wurden am Computer präsentiert. Die abhängigen Variablen wurden über multiple choice-Tests erhoben: Jede Stunde endete mit neun Fragen (je drei Wiederholungs-, Verständnis- und Problemlöse-Items). Zwei Wochen nach der letzten Stunde wurde ein Erinnernstest durchgeführt, der je 18 Wiederholungs-, Verständnis- und ProblemlöseItems umfasste. Am Ende jeder Stunde bearbeiteten die Versuchspersonen zudem noch ein Problem, das zu Beginn der Stunde benutzt wurde, um ihnen ihr Vorwissen zu entlocken. Die Lösung stellte natürlich die „finale Idee“ dar. In der CONTACT-Gruppe begann jede Stunde mit einem konkreten Problem (oder einer Frage), das von den Schülern gelöst werden sollte, um das Vorwissen zu eruieren (1. Schritt). Ein Beispiel: „Als Kolumbus 1492 Segel setzte, blies ihn der Wind nicht geradewegs auf den Äquator zu. Stattdessen wurde er in einer Rechtskurve nach Amerika geblasen. Kannst Du das erklären?“ Die Versuchspersonen konnten zwischen sechs Antworten wählen und mussten ihre Wahl begründen. Die Distraktoren enthielten Fehlkonzepte, die typischerweise in diesem Alter gemacht werden. Dann wurde in der Lektion die neue Information präsentiert (2. Schritt). Danach wurde die Ausgangsfrage noch einmal gestellt; wiederum mussten die Schüler eine Antwort auswählen und diese begründen (3. Schritt). Danach wurde ihnen ein neues, aber verwandtes Problem gegeben, das sie mit ihrem neu erworbenen Wissen lösen konnten (4. Schritt). Zuletzt sollten sie ihr neu erworbenes Wissen bewerten und mit dem Vorwissen vergleichen (5. Schritt). Gaben die Schüler im 3. Schritt eine falsche Antwort, wurden sie aufgefordert, die Lektion noch einmal durchzulesen. Zu jedem Schritt war Information über seine Relevanz (Warum ist das wichtig?) und seine Durchführung (Wie macht man das?) abrufbar. In einigen Stunden wurde diese Warum- und Wie-Information allen Schülern präsentiert. In anderen Stunden konnten die Schüler diese Information nach eigenem Gusto abrufen. Alle fünf Schritte wurden auch metaphorisch visuell dargestellt.

BIEMANS fand in einer Studie, dass die CONTACT-Strategie bei allen Tests (Wiederholung, Verständnis und Problemlösen) zu signifikant besseren Ergebnissen führte als bei einer Gruppe, deren Mitglieder nur den ersten und den letzten Schritt der Strategie durchführten und weder Feedback noch Zusatzinformationen erhielten. In einer zweiten Studie traten signifikante Unterschiede zwischen Schülern mit der CONTACT-Strategie, Schülern mit dem Erster-undLetzter-Schritt-Ansatz, und Schülern einer Kontrollgruppe (ohne Hilfeangebote) zutage. In einer dritten Studie zeigte BIEMANS, dass es möglich ist, Schülern beizubringen, die CONTACTStrategie eigenständig zu nutzen. Den Schülern wurde beigebracht, wie sie die CONTACT-Schritte selbstständig durchführen konnten; dann wurde die bereitgestellte Hilfe nach und nach 
ausgeblendet. Auch hier lernten die CONTACT-Schüler besser. Offenbar ist es möglich, Schüler anzuleiten, beim Lernen die Grundsätze der CONTACT-Strategie zu verwenden.

\section{Vierte Kluft: Anwenden von Kompetenzen}

Die nächste Kluft wird beim Anwenden von Kompetenzen deutlich (Abbildung 5; Kluft 4).

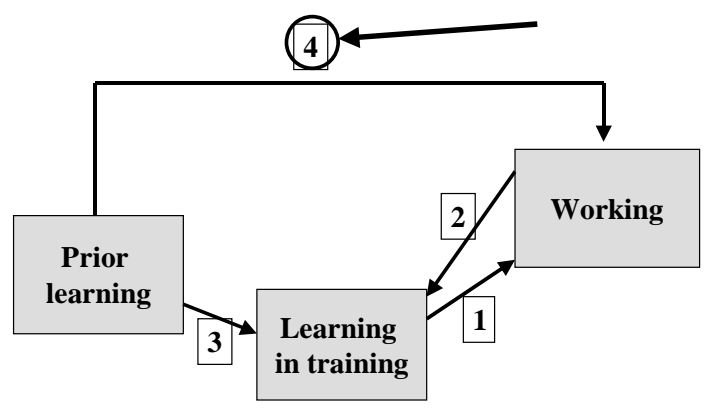

Abb. 5: Anwenden von Kompetenzen (Kluft 4).

Menschen setzen bei der Arbeit nicht alle ihre Kompetenzen ein. Organisationen neigen dazu, die Kompetenzen ihrer Beschäftigten zu unterschätzen (SENGE, 1991). Offizielle Qualifikationen, also jene Anforderungen des Arbeitsmarktes, die in Zeugnissen und anderen offiziellen Dokumenten niedergelegt sind, sind nicht das, was die Organisationen wirklich brauchen. Damit ist eine weitere Kluft skizziert zwischen dem, was Menschen lernen, und dem, was sie in der Arbeit zu leisten haben.

Es liegt Evidenz vor (DOORnBOS \& KRAK, 2001), dass das, was bei der Arbeit gelernt wird, oft implizit bleibt. Dies wird erkennbar, wenn Beschäftigte versuchen, darüber zu sprechen. Als DOORNBOS und KRAK Polizeibeamte befragten, was sie am Arbeitsplatz gelernt hätten, konnten sie kaum etwas berichten. Im weiteren Verlauf der Studie zeigte sich aber, dass die Frage nach dem „Lernen“ ein Holzweg der Forscher war. Beim Wort „Lernen“ gerieten die Befragten auf eine falsche Fährte - sie versuchten, sich an besuchte Kurse zu erinnern, an gelesene Bücher, an genossenes Coaching usw. Wurde das Wort „Lernen“ vermieden und hatten sie stattdessen über Veränderungen in ihrer Kompetenz zu berichten (siehe nächsten Absatz), begannen sie zu erkennen, dass sie viel in und von ihrer Arbeit gelernt hatten. Durch den Hinweis auf konkrete Veränderungen in Arbeitsprozessen oder -ergebnissen wurden sie sich ihrer eigenen Lernprozesse bewusst. Und nachdem ihnen klar war, was sie gelernt hatten, begannen sie darüber zu sprechen, wie sie es gelernt hatten.

Da ein großer Prozentsatz unseres Lernens implizit abläuft (SIMONS, VAN DER LINDEN \& DUFFY, 2000), faszinierte uns die Untersuchung dieses impliziten Lernens. Seither eruieren wir, wie man an implizite Lernergebnisse (und damit auch an implizite Lernprozesse) herankommt.

Zunächst ist es wichtig zu realisieren, dass es weder möglich noch erstrebenswert ist, alles implizite Lernen explizit zu machen. Manchmal ist es sogar besser, implizites Lernen nicht explizit zu machen. Wie NONAKA und seine Kollegen (z.B. VON KrOGH, ICHIJO \& NonAKA, 2000) zeigten, gibt es auch implizite Formen des Austauschs. Gerade durch informelle Prozesse und in informellen Settings entsteht oft ein Gefühl "geteilter Kompetenz" (NONAKA, REINMOELLER \& SENOO, 1998).

Will man Lernen explizit machen, gibt es verschiedene Möglichkeiten. Zunächst ist zwischen „reflection-in-action“ und „reflection-on-action“ zu unterscheiden. Unter der Bedingung ,inaction" sind unsere impliziten Kompetenzen - auch wenn sie uns nicht bewusst sind - für 
Andere sichtbar. Indem er Andere „in-action“ beobachtet, kann ein geschulter Beobachter zu Grunde liegende Kompetenzen erschließen. Durch den Einsatz von Interviews vor und nach den beobachteten Handlungen können Menschen lernen, die Handlungen zu reflektieren, um sich der eigenen impliziten Kompetenzen (Wissen, Fähigkeiten, Einstellungen) bewusst zu werden. Unsere Erfahrung zeigt, dass reflexive Praxis im Sinne von SCHÖN (1987) eine sehr wirkungsvolle Methode sein kann. Auf diesem Hintergrund konnte KLARUS (1998) eine effektive Lernmethode ersinnen und analysieren, die aus einer Kombination von Interviews vor und nach den relevanten Arbeitshandlungen mit Beobachtungen besteht.

„Reflektion-on-action“ ist wohl weniger akkurat, aber dennoch sehr informativ. Um es durchzuführen, bittet man entweder andere Personen, das eigene Lernen zu reflektieren, oder man macht es selbst. Klienten und Kollegen können oft ein hervorragendes Gespür für die besonderen Kompetenzen der Personen haben, mit denen sie zu tun haben. Sie können daher hilfreich dabei sein, sich des eigenen impliziten Lernens gewahr zu werden. Man kann Klienten einfach wie folgt um Feedback bitten: „Was zeichnet Ihrer Erfahrung nach meine Art des Arbeitens aus?" Daneben kann auch die $360^{\circ}$-Feedback-Methode wirkungsvoll sein. Der Einsatz reflexiver Methoden ist aber wohl einfacher (sei es individuelle oder kollektive Reflexion).

Die erste Technik, Lernergebnisse durch individuelle Reflexion explizit zu machen, stammt von ERAUT (1998) und Kollegen. Anstatt direkt nach den Lernergebnissen zu fragen, fragten sie Polizisten und Krankenschwestern, was sich in ihrer Arbeit geändert hatte. „In welcher Hinsicht unterscheidet sich Ihre heutige Arbeit davon, wie sie vor zwei [fünf usw.] Jahren war?““ „Was sagt Ihnen das, was Sie jetzt wissen bzw. tun können, darüber, was Sie damals nicht wussten bzw. tun konnten?" Bei einer anderen Technik wird nach einem idealen Berufsangehörigen gefragt, z.B.: „Wie führt ein idealer Zugschaffner [Manager usw.] seine Arbeit aus?" Wir haben es oft erlebt, dass Beschäftigte sehr konkrete Vorstellung von idealen Modellen haben. Ein Folgefrage könnte sein: „In welcher Hinsicht sind Sie selbst schon ein idealer Zugschaffner [Manager usw.]?“ Dies führt fast automatisch dazu, dass nach Erklärungen für die eigenen Lernergebnisse und für Unterschiede im Vergleich zum Ideal gesucht wird. Manchmal werden dabei auch Brüche zwischen der eigenen gegenwärtigen Arbeitsweise und dem Ideale entdeckt und wird damit der Weg für innovative Lerngelegenheiten gebahnt. Beispielsweise trafen wir einen Manager, der sein Ideal als jemanden beschrieb, der permanent in einem Netzwerk mit allen Managern der Organisation informell zusammenarbeitet. Durch die Beschreibung wurde ihm klar, dass er selbst nicht so arbeitete. Menschen nach dem Unterschied zwischen ihrem Idealbild und den eigenen impliziten und expliziten Kompetenzen zu fragen, kann also dazu führen, dass bis dato erreichte Lernergebnisse bewusst werden, aber auch notwendige künftige Lernprozesse. Eine dritte, oft verwendete Technik ist die critical incident method. Dabei sollen sich Personen an kritische Ereignisse erinnern. Dies dient als Ausgangspunkt, über zu Grunde liegende Kompetenzen nachzudenken. Diese Technik ähnelt den pretty good practices-Ansatz von MARSICK (2001), in dem die Beschäftigten über Situationen reden, die sie ,ziemlich gut" bewältigt haben. MARSICK legt Wert darauf, nicht nach der „besten“ Praxis zu fragen, weil dies zu viel Leistungsdruck ausüben würde. Unsere Erfahrungen zeigen, dass es bei dieser Methode wichtig ist, über positive Erfahrungen zu sprechen, nicht über eigene Fehler. Positive kritische Ereignisse und pretty good practices erhöhen die Bereitschaft (und schwächen Abwehrmechanismen), um Details über Lernergebnisse und -prozesse nachzuspüren. Verwandt mit dieser Technik ist die story telling-Technik, bei der Anekdoten und Geschichten über die eigene Arbeit erzählt werden. Der einzige Unterschied ist, dass die Perspektive des Geschichten-Erzählers dazu führt, dass Details in der Beschreibung der Umstände und der Ereignisse aus einer gewissen Distanz heraus erfolgen, wenn die Personen über sich selbst in der dritten Person sprechen.

Eine weitere Technik, die von MARSICK (2001) beschrieben wird, ist die des walking in the shoes of the client. Die Versuchspersonen werden dabei aufgefordert, die Perspektive eines ihrer Klienten einzunehmen und ihre eigenen Kompetenzen durch die Brille dieses fiktiven Klienten zu betrachten. Ein letztes Beispiel, das von MARSICK (2001) beschrieben wird, ist schließlich der multiple intelligences-Ansatz, bei dem versucht wird, sich durch den Einsatz von Bildern, 
Zeichnungen, Metaphern, Bewegungen usw. darüber klar zu werden, was man implizit gelernt hat. Natürlich könnte man sich noch viele weitere Techniken denken, wie etwa „Andere unterrichten“, „In die Zukunft schauen“, „In heteronomen Gruppen arbeiten“ oder „Über Veränderungen und Verbesserungen nachdenken“. All diese Methoden haben gemeinsam, dass sie von einer konkreten Situation oder Erfahrung ausgehen und erst dann zu Lernergebnissen oder Kompetenzen gelangen.

\section{Fünfte Kluft: Lernen am Arbeitsplatz}

Die fünfte Kluft ist die zwischen Arbeit und Lernen bei der Arbeit; hier handelt es sich also um das Lernen am Arbeitsplatz (Abbildung 6; Kluft 5).

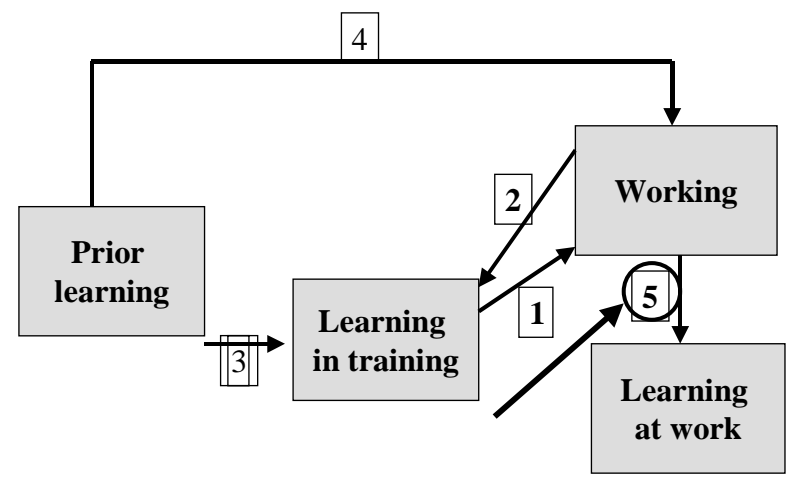

Abb. 6: Lernen am Arbeitsplatz (Kluft 5).

Man könnte meinen, dass es hier gar keine Kluft gibt (und einige Autoren nehmen dies auch an), weil Arbeit und Lernen am Arbeitsplatz so eng verwandt sind. Und doch gibt es diese Kluft. Natürlich lernt man immer etwas am Arbeitplatz, aber eben nicht auf die beste Art und Weise; es ist daher nicht so, dass das Lernen am Arbeitplatz nicht verbessert werden könnte. Ein Phänomen, dem wir begegneten, war, dass Menschen viel mehr am Arbeitsplatz lernen, als sie selbst wahrhaben (DOORNBOS \& KRAK, 2001). Sie lernen aber nur selten bewusst am Arbeitsplatz. Implizites Lernen ist vielleicht für annähernd 80\% der Kompetenzentwicklung in der Arbeit verantwortlich. Des Weiteren kann Lernen am Arbeitsplatz verschiedenerlei stattfinden, z.B. durch implizites Lernen, selbstreguliertes Lernen oder Lernen durch explizite Anweisung eines Trainers oder Mentors. Beim impliziten Lernen sind sich die Menschen nicht bewusst darüber, dass sie lernen. Lernen ist hier ein Nebeneffekt von Arbeiten. Beim selbstregulierten Lernen planen und organisieren Menschen ihr Lernen explizit und bewusst, wenngleich größtenteils im Arbeitskontext. Bei der dritten Lernart planen und organisieren Dritte (Manager, Coaches, Trainer) explizit und bewusst (das gilt nicht immer auch für die Lernenden) das Lernen. Selbstreguliertes Lernen ist eher die Ausnahme. Insgesamt gäbe es am Arbeitsplatz viel mehr Gelegenheit, Mentoring und Coaching einzusetzen.

Pädagogen bevorzugen es, Lernen explizit zu organisieren (das gilt in Schulen genauso wie am Arbeitsplatz), und zwar bezogen sowohl auf die Lernergebnisse als auch auf die Lernprozesse. Mit „Pädagogisieren“ meinen wir daher, implizites durch explizites Lernen ersetzen zu wollen. Dies ist oft ein Fehler, wenn versucht wird, Lernen da zu planen und zu organisieren, wo es besser im Arbeitszusammenhang spontan auftauchen sollte. Es gibt mehrere Gründe, wieso Pädagogisieren ein Fehler sein kann. (1) Ein großer Teil des Lernens am Arbeitsplatz ist, wie bereits erwähnt, implizit. (2) Oft mögen es Menschen, neue Lösungen und Handlungsmöglichkeiten selbst (also: implizit) zu lernen, aber stören sich an expliziten Lernweisen. (3) Es kann zuviel Aufmerksamkeit auf das Lernen statt auf die Arbeit gelenkt 
werden. (4) Die Motivation der Lernenden kann ruiniert werden. Somit sollten, bevor pädagogisiert wird, drei Fragen gestellt werden:

(1) Was resultiert aus der Pädagogisierung spezifischer Arbeitssituationen?

(2) Was wird gewonnen, wenn implizites durch explizites Lernen ersetzt wird?

(3) Können wir die Arbeitsumgebung so umgestalten, dass die Wahrscheinlichkeit erhöht wird, dass implizites Lernen stattfindet?

In vielen Fällen ist es vermutlich besser, Wege zu finden, Beschäftigten und Managern dabei zu helfen, Arbeit so umzugestalten, dass mehr implizites Lernen stattfinden kann. Ist es möglich, das zu machen, ohne Lernen zu expliziter zu machen? Wenn ja: Wie? Die Studien von ONSTENK (1997) und KWAKMAN (1999) lehren, dass die Konzentration auf sechs Merkmale von Arbeitsprozesses und der Arbeitsumgebung dies ermöglicht: (1) Variation, (2) Verantwortung, (3) Feedback, (4) Reflexion, (5) Innovation/Experimentieren und (6) Aufbau einer Vision.

Diese sechs Merkmale können von Managern durchaus reorganisiert werden (Zeit für Reflexion geben, Feedback organisieren, Autonomie geben, Innovationen und Experimente planen usw.), allerdings genauso gut auch von den Lernenden selbst. (In letzterem Fall kommen wir in das Reich selbstregulierten Lernens.) Lernende können versuchen, nach Feedbackgelegenheiten zu suchen, Zeit für Reflexion zu reservieren, nach Variation zu suchen, für Innovation offen zu sein usw. All dies kann individuell oder kollaborativ organisiert mit Kollegen, Trainern, Managern oder Klienten organisiert sein. Jede dieser Personengruppen kann eine neue Perspektive aufzeigen und damit zum impliziten Lernen beitragen.

\section{Sechste Kluft: Transfer vom Training zum weiteren Lernen am Arbeitsplatz}

Die letzte Kluft bezeichnet den Transfer vom Training zum weiteren Lernen am Arbeitsplatz (Abbildung 7; Kluft 6).

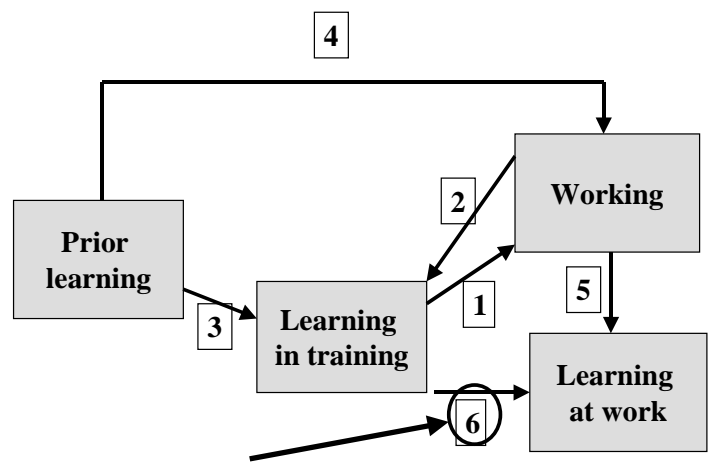

Abb. 7: Transfer vom Training zum weiteren Lernen am Arbeitsplatz (Kluft 6).

Ideale Lernumgebungen verhelfen Lernenden nicht nur zu guten Lernprozesse und -ergebnissen, sondern bereiten sie auch auf späteres Lernen vor (CANDY \& CREBERT, 1990). In ihrer Dissertation zeigte TEURLINGS (1993) dies exemplarisch in einer Studie, in der erwachsene Lerner über mehrere Sitzungen hinweg lernten, mit WordPerfect $5.1 \mathrm{zu}$ arbeiten. In der Kontrollgruppe wurden die Stunden wie in normalen Trainings organisiert. In der Experimentalgruppe lernten die Versuchspersonen nicht nur, mit WP zu arbeiten, sondern auch, wie sie selbst mehr über WP lernen konnten. Dazu gab es zunächst ein Video, das demonstrierte, dass das Lernen von WP nichts mit körperlichem Geschick zu tun hat (viele Lernende glaubten, bei dem Kurs ginge es um etwas Ähnliches wie Schreibmaschine schreiben), sondern vielmehr damit, zu lernen, „wie ein Computer zu denken“. Zweitens wurde eine Analogie zu einem 
komplexen Gebäude aufgebaut: Die Lernenden erfuhren, wie man sich im Computer zurechtfindet, wohin man auf dem Bildschirm sehen muss, wie man herausfindet, wo im Programm man sich gerade befindet, wie das Programm prinzipiell aufgebaut ist usw. Drittens erfuhren die Lernenden den Umfang mit verschiedenen Hilfefunktionen (kleines Handbuch, Online-Unterstützung). Viertens wurden sie motiviert, zu experimentieren und neue Dinge auszuprobieren, und erfuhren so, dass nichts Ernsthaftes passieren kann, solange man nicht ein, zwei bestimmte Fehler macht (wie z.B. „,format c:"). Fünftens beschränkte sich das Training auf grundlegende Fähigkeiten und Prozeduren, um Zeit für die Förderung der Lernfähigkeit zu schaffen. Beispielsweise wurden den Lernenden nur gezeigt, wie sie einen Text unterstreichen konnten; zudem wurden sie ermutigt, selbst herauszufinden, wie man ähnliche Dinge macht (z.B. einen Text fett drucken).

Es zeigte sich, dass die Lernenden der Experimentalgruppe nicht nur mehr vom Unterrichteten lernten als die der Kontrollgruppe, sondern, was viel wichtiger ist, sie lernten auch viel schneller und effektiver, sich neue Aspekte von WP selbst zu erarbeiten. Dieser Effekt war sowohl direkt nach dem Training als auch noch sechs Wochen später beobachtbar.

\section{Schlussfolgerungen}

Hoffentlich wurde deutlich, dass Transfer viel mehr umfasst als Trainingstransfer. Die anderen fünf Transferarten scheinen mindestens ebenso wichtig zu sein. Sie werden nur selten als Transfer konzipiert, sondern firmieren unter anderen Überschriften (z.B. „Lernen lernen“, „Fehlkonzepte"). Alle sechs Phänomene haben aber so viele Gemeinsamkeiten, dass es vernünftig ist, sie alle als „Transfer“ zu bezeichnen. Vor allem gibt es drei große Gemeinsamkeiten:

(1) In allen Fällen geht es um eine Balance zwischen Kontextualisierung und Dekontextualisierung. Es ist sowohl nötig, eine Verbindung zum Kontext, zur Arbeitskultur und zur community of practice herzustellen, als auch das Lernen zu dekontextualisieren. Kontextualisierung führt eher $\mathrm{zu}$ nahem Transfer, Dekontextualisierung eher zu weitem Transfer.

(2) In allen Fällen wird von einer Situation auf einer andere verallgemeinert. Generalisierung impliziert eher weiten Transfer.

(3) In allen Fällen spielen Metakognition und Reflexion, Variation, situationale Bedingungen und Anwendungsmöglichkeiten eine große Rolle.

\section{Literaturverzeichnis}

Ali, K. (1990). Instructiestrategieën voor het activeren van preconcepties [Unterrichtsstrategien zur Aktivierung von Vorwissen] Unveröff. Diss., Universität Tilburg.

Baldwin, T. T. \& Ford, J. K. (1988). Transfer of training: A review and directions for future research. Personnel Psychology, 41, 63-105.

Biemans, H. J. A. (1997). Fostering activation of prior knowledge and conceptual change. Unveröff. Diss., Universität Nijmegen.

Biemans, H. J. A. \& Simons, P. R.-J. (1995). How to use preconceptions? The contact strategy dismantled. European Journal of Psychology of Education, 10, 243-259.

Biemans, H. J. A. \& Simons, P. R.-J. (1996). A computer-assisted instructional strategy for promoting conceptual change. Instructional Science, 24, 157-176.

Brinkerhoff, R. O. \& Gill, S. J. (1992), Managing the total quality of training. Human Resource Development Quarterly, 2, 121-131. 
Broad, M. L. \& Newstrom, J. W. (1992). Transfer of training. Action packaged strategies to ensure high payoff from training investments. Reading: Addison-Wesley.

Burke, L. (1997). Improving positive transfer: A test of relapse prevention training on transfer outcomes. Human Resource Development Quarterly, 8, 115-128.

Candy, P. C. \& Crebert, R. G. (1990, Juli). Teaching now for learning later: The transfer of learning skills from the academy to the workplace. Vortrag auf der " $\mathrm{s}^{\text {th }}$ Australian Learning and Language Conference", Brisbane, Australien.

Claas, L., Pouwer, M. \& Thijssen, J. G. L. (1986). Transfer in het kader van bedrijfsopleidingen. [Transfer im Kontext industrieller Trainings] 's Gravenhage: ROI/CIVOB.

Cormier, S. M. \& Hagman, J. D. (1987). Introduction. In S. M. Cormier \& J. D. Hagman (Hrsg.), Transfer of learning. Contemporary research and applications (S. 1-8). San Diego: Academic Press.

Doornbos, A. J. \& Krak, A. J. A. (2001, Januar). Learning processes and outcomes at the workplace: A qualitative study. Vortrag auf der "Second Conference on HRD Research and Practice Across Europe", Enschede, Niederlande.

Eraut, M. R. (1998). Development of knowledge and skills in employment. Brighton: University of Sussex Institute of Education.

Gaines-Robinson, D. \& Robinson, J. C. (1989). Training for impact. San Francisco: Jossey-Bass.

Geelen, M. \& Hendriks, E. (1998). Projectmanagement Achmea. Unveröff. Master Thesis, Universität Nijmegen.

Hewson, P. W. \& Hewson, M. G. A. (1984). The role of conceptual conflict in conceptual change and the design of science instruction. Instructional Science, 13, 1-13.

Kersten, J. (1990). Transfer-bevorderende activiteiten in de opleiding tot psychiatrisch verpleegkeundige. [Transferförderliche Aktivitäten im Training von Pflegepersonal in der Psychiatrie] Unveröff. Master Thesis, Universität Nijmegen.

Klarus, R. (1998). Competenties erkennen. Een studie naar modellen en procedures voor leervegonafhankelijke beoordeling van beroepscompetenties. [Kompetenzen erkennen: Eine Studie über Modelle und Vorgehensweisen bei der lernwegunabhängigen Evaluation beruflicher Kompetenzen] Unveröff. Diss., Universität Nijmegen.

Kwakman, C. H. E. (1999). Leren van docenten tijdens de beroepsloopbaan. [Lernprozesse von Lehrern in ihrem Berufsleben] Unveröff. Diss., Universität Nijmegen.

Lether, E. B. (1997). Transfer-effect van een bedrijfsopleiding. [Transfereffekt eines betrieblichen Trainings] Unveröff. Master Thesis, Universität Nijmegen.

Marsick, V. (2001, Januar). Informal strategic learning in the workplace. Vortrag auf der "Second Conference on HRD Research and Practice Across Europe", Enschede, Niederlande.

Marx, R. (1992). Relapse prevention for managerial training: A model for maintenance of behavior change. Academy of Management Review, 7, 433-441.

Nonaka, I., Reinmoeller, P. \& Senoo, D. (1998). The 'ART' of knowledge: Systems to capitalize on market knowledge. European Management Journal, 16, 673-684.

Nussbaum, J. \& Novick, S. (1982). Alternative frameworks, conceptual conflict and accommodation: Towards a principled teaching strategy. Instructional Science, 11, 183-200.

Onstenk, J. H. A. M. (1997). Lerend leren werken. Brede vakbekwaambeid en de integratie van leren, werken en innoveren. [Lernen, arbeiten zu lernen. Unspezifische Kompetenzen und die Integration von Lernen, Arbeiten und Innovation] Unveröff. Diss., Universität Nijmegen.

Pea, R. D. (1987). Socializing the knowledge transfer problem. International Journal of Educational Research, 11, 639-663.

Prawat, R. S. (1989). Promoting access to knowledge, strategy and disposition in students: A research synthesis. Review of Educational Research, 59, 1-41.

Schön, D. A. (1987). Educating the reflective practitioner: Toward a new design for teaching and learning in the professions. San Francisco: Jossey-Bass.

Senge, P. (1991). The fifth discipline. New York: Double Day.

Siegler, R. S. (1983). Five generalizations about cognitive development. American Psychologist, 38, 263-277.

Simons, P. R.-J. (1990). Transfervermogen. [Transferfähigkeit]. Nijmegen: Quick Print.

Simons, P. R.-J. (1999). Transfer of learning: Paradoxes for learners. International Journal of Educational Research, $31,577-589$ 
Simons, P. R.-J., van der Linden, J. \& Duffy, T. (2000). New learning: Three ways to learn in a new balance. In P. R.-J. Simons, J. van der Linden \& T. Duffy (Hrsg.), New learning (S. 1-20). Dordrecht: Kluwer.

Strike, K. A. \& Posner, G. J. (1985). A conceptual change view of learning and understanding. In L. H. T. West \& A. L. Pines (Hrsg.), Cognitive structure and conceptual change (S. 211-231). Orlando: Academic Press.

Ter Heijne, B. \& Termote, A. (1990). Het geleerde in praktijk gebracht: Een onderzoek naar de invloed van transferbevorderende maatregelen in de praktijk-opleiding tot radio-diagnostisch laboratory worker. [Das Gelernte in Praxis umsetzen: Forschung über den Einfluss transferfördernder Maße beim Training von Arbeitern in radio-diagnostischen Laboratorien] Unveröff. Master Thesis, Universität Nijmegen.

Teurlings, C. C. J. (1993). Leren tekstververken: Een nieuw perspectief. [Lernen, Textverarbeitung zu benutzen: Eine neue Perspektive] Unveröff. Diss., Universität Tilburg.

Von Krogh, G., Ichijo, K. \& Nonaka, I. (2000). Enabling knowledge creation. Oxford: Oxford University Press. 